\title{
The relative contributions of temperature and volume to structural relaxation of van der Waals molecular liquids
}

\author{
M. Paluch \\ Naval Research Lab, Chemistry Division, Washington, DC 20375-5342 \\ and Institute of Physics, Silesian University, Uniwersytecka 4, 40-007 Katowice, Poland \\ C. M. Roland ${ }^{\text {a) }}$ and R. Casalini \\ Naval Research Lab, Chemistry Division, Washington, DC 20375-5342 \\ G. Meier \\ Forschungszentrum Juelich Institute of Solid State Research Postfach 1913, 52428 Juelich, Germany
}

A. Patkowski

Institute of Physics, A. Mickiewicz University, Umultowska 85, 61-614 Poznan, Poland

(Received 3 September 2002; accepted 19 December 2002)

\begin{abstract}
Pressure-volume-temperature measurements were carried out on two van der Waals liquids, 1,1' '-bis(p-methoxyphenyl)cyclohexane (BMPC) and 1,1'-di(4-methoxy-5methylphenyl)cyclohexane (BMMPC). In combination with dielectric spectroscopy results, the relative contribution of temperature and density to the structural relaxation times were quantified. We find that the ratio of the isobaric expansion coefficient $\left[-\rho^{-1}(\partial \rho / \partial T)_{P}\right.$, where $\rho$ is mass density and $T$ is temperature, evaluated at $P=0.1 \mathrm{MPa}$ to the coefficient of isochronal expansivity $\left[-\rho^{-1}(\partial \rho / \partial T)_{\tau}\right.$, evaluated at $\left.\tau=1 \mathrm{~s}\right]$ equals 0.58 and 0.72 for BMPC and BMMPC, respectively. This indicates that density exerts more influence on the structural relaxation times than does thermal energy. Corroborating this finding, the ratio of the isochoric activation energy to the activation energy at constant pressure is determined to be ca. 0.4 at ambient pressure for both glass formers. The prevalence of density over thermal energy is contrary to prevailing ideas concerning the dynamics of supercooled liquids, and must be taken into account in developing models of the glass transition. (C) 2003 American Institute of Physics. [DOI: 10.1063/1.1545449]
\end{abstract}

\section{INTRODUCTION}

At sufficiently high temperatures, when intermolecular cooperativity becomes negligible, the time scale characterizing structural relaxation of glass-formers follows the Arrhenius law. However, for most polymers and molecular liquids, at lower temperatures, the temperature dependence of the relaxation times, $\tau$, and viscosity increase in a non-Arrhenius fashion (referred to as super-Arrhenius behavior). This slowing down of molecular rearrangements in supercooled liquids is brought about by both a decrease of thermal energy and an increase in molecular crowding (packing density). To evaluate the relative contributions of these two effects requires measurements of both the temperature and density dependence of $\tau$. In practice, this is accomplished by carrying out two types of experiments, measurement of the combined temperature and pressure dependences of the structural relaxation time (or viscosity), in combination with a determination of the volume as a function of $T$ and $P$.

Such experiments are of great importance, since the roles of thermal energy and free volume fluctuations are essential to formulating a realistic theory of the slow dynamics of glass formers, and to provide critical tests for existing

\footnotetext{
a) Author to whom correspondence should be addressed. Electronic mail: roland@nrl.navy.mil
}

models. Presently there are two contrary points of view: Energy landscape models, ${ }^{1-5}$ descendants from the early work of Eyring ${ }^{6}$ and Frenkel, ${ }^{7}$ focus on thermally activated transport over potential barriers. The role of density is limited to its effect on the topology of the energy landscape. The alternative approach, prevalent for polymers, ${ }^{8}$ emphasizes the congested nature of the supercooled state ("crowding couplings"), and the manner in which the cooperative dynamics are governed by free volume and its fluctuations. ${ }^{9-12}$ In this view point, temperature alters the free volume, but the effect of thermal energy is not directly addressed.

Alba-Simionesco, Kivelson, and co-workers, ${ }^{13,14}$ from analysis of experimental and simulation data, argued that the super-Arrhenius behavior near the glass transition at ambient pressure is governed overwhelmingly by temperature. This suggestion supports models that focus on activated dynamics, with free volume concepts being neglectable. Nevertheless, free volume models for supercooled liquids have a strong intuitive appeal, and the use of positron annihilation lifetime spectroscopy (PALS) $)^{15-17}$ to measure the unoccupied volume contributes to the popularity of this approach. Modifications to incorporate temperature-dependent parameters into a free volume model have been proposed. ${ }^{18,19}$

Recently, we determined that for a number of low molecular weight glass-forming liquids, the relative magnitudes 


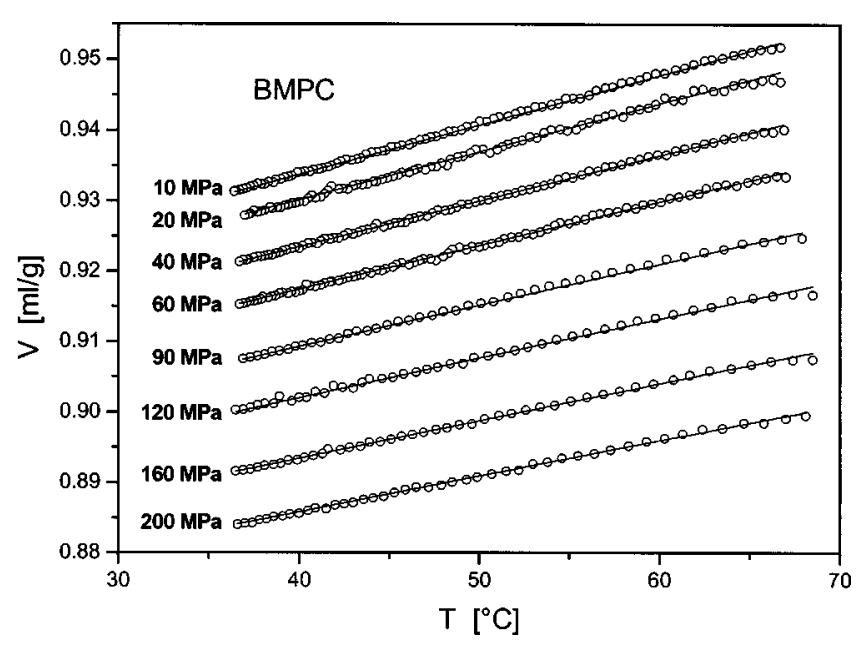

FIG. 1. Temperature dependence of the specific volume of BMPC, measured at the indicated pressures. The solid lines represent Eq. (1), using the parameters listed in Table I.

of the effects of thermal and free volume fluctuations on the structural relaxation times are quite comparable. ${ }^{20-23}$ In light of these findings, the idea that temperature is the dominant control variable for structural relaxation at ambient pressure cannot be generally true for glass-formers. Quite the contrary, it appears to hold only for a limited group of liquids, characterized by strong specific interactions (e.g., hydrogen bonding), such as glycerol ${ }^{14}$ and sorbitol. ${ }^{24}$

In this article we use equation of state measurements to further demonstrate that temperature does not govern decisively the super-Arrhenius behavior of all glass-forming liquids. As detailed below, for two well-known van der Walls liquids, 1,1'-bis(p-methoxyphenyl)cyclohexane (BMPC) and 1,1'-di(4-methoxy-5-methylphenyl)cyclohexane (BMMPC), volume exerts a stronger effect on the structural relaxation times than does thermal energy. In these particular glass formers, the volume contribution is the highest reported in the literature.

\section{EXPERIMENT}

The synthesis of the BMPC and BMMPC can be found elsewhere. ${ }^{25}$ Note that these compounds are also referred to in the literature as bis-phenol-C-dimethylether (BCDE) and bis-kresol-C-dimethylether (BKDE), respectively.

The PVT experiments employed a Gnomix instrument. ${ }^{26}$ The sample (typically $1.5 \mathrm{~g}$ ) was wrapped in nickel foil, then immersed in mercury. Measurements of volume changes were carried out isothermally as a function of pressure (up to $200 \mathrm{MPa}$ ), at each temperature. The lower limit for the latter

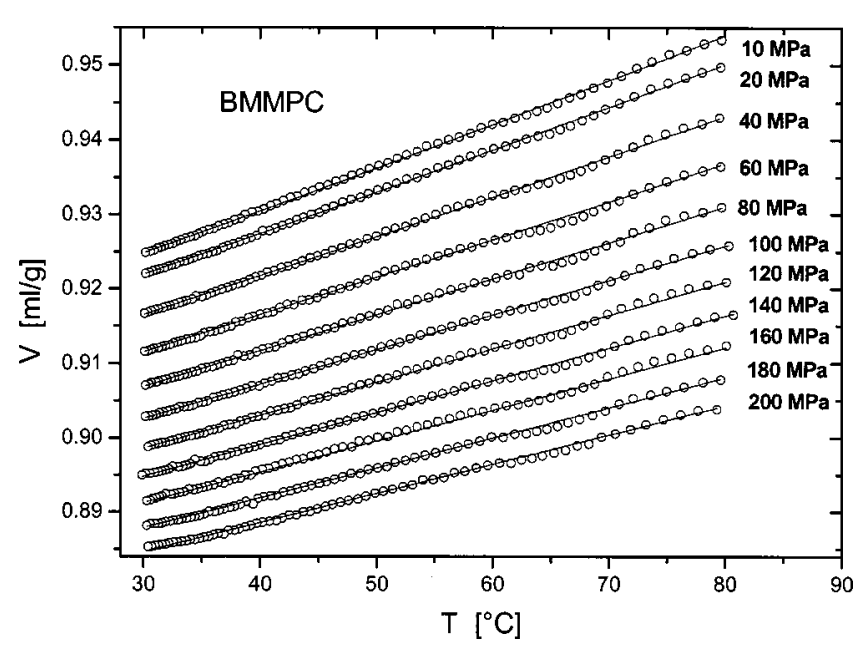

FIG. 2. Temperature dependence of the specific volume of BMMPC, measured at the indicated pressures. The solid lines represent Eq. (1), using the parameters listed in Table I.

was $293 \mathrm{~K}$; thus, the data for the supercooled liquids does not reach the glass temperature (equal to 240 and $261 \mathrm{~K}$ for $\mathrm{BMPC}$ and BMMPC, respectively ${ }^{27}$ ).

\section{RESULTS}

Figures 1 and 2 show the PVT curves for BMPC and BMMPC, respectively. Above $T_{g}$, the volume data conform well to the Tait equation ${ }^{28}$

$$
V(T, P)=V(T, 0)[1-0.0894 \ln (1+P / B(T))]
$$

with $\quad V(T, 0)=\nu_{0}+\nu_{1} T+\nu_{2} T^{2}, \quad$ and the $B(T)=b_{0}$ $\times \exp \left(-b_{1} T\right)$. The values of these parameters, obtained by simultaneously fitting the curves in Figs. 1 and 2, are given in Table I.

Using Eq. (1), the structural relaxation times measured dielectrically for $\mathrm{BMPC}^{29}$ and $\mathrm{BMMPC}^{30}$ can be expressed as a function of volume. These curves, shown in Figs. 3 and 4 , demonstrate clearly that the relaxation times are not uniquely defined by the volume. Such a result does not a priori rule out free volume models for the glass transition, since they posit fractional free volume as the governing parameter. ${ }^{8}$ However, the isobaric data exhibit a steeper change, reflecting the direct role of thermal energy, beyond any contribution from volume changes per se.

A measure of the relative magnitudes of the temperature and volume contributions to the relaxation times can be gleaned from comparison of the temperature dependence of $\tau_{\alpha}$ for experiments at constant volume (isochoric) versus at

TABLE I. Equation of state parameters.

\begin{tabular}{cccccc}
\hline \hline & $\begin{array}{c}\nu_{0} \\
(\mathrm{ml} / \mathrm{g})\end{array}$ & $\begin{array}{c}\nu_{1} \\
(\mathrm{ml} / \mathrm{g} \mathrm{C})\end{array}$ & $\begin{array}{c}\nu_{2} \\
\left(\mathrm{ml} / \mathrm{g} \mathrm{C}^{2}\right)\end{array}$ & $\begin{array}{c}b_{0} \\
(\mathrm{MPa})\end{array}$ & $\begin{array}{c}b_{1} \\
\left(\mathrm{C}^{-1}\right)\end{array}$ \\
\hline BMPC & $0.716 \pm 0.006$ & $7.2 \pm 1 \times 10^{-4}$ & 0 & $201 \pm 2$ & $4.31 \pm 0.10 \times 10^{-3}$ \\
BMMPC & $0.690 \pm 0.005$ & $5.5 \pm 0.1 \times 10^{-4}$ & $4.5 \pm 0.5 \times 10^{-7}$ & $235 \pm 3$ & $4.96 \pm 0.01 \times 10^{-3}$ \\
\hline \hline
\end{tabular}




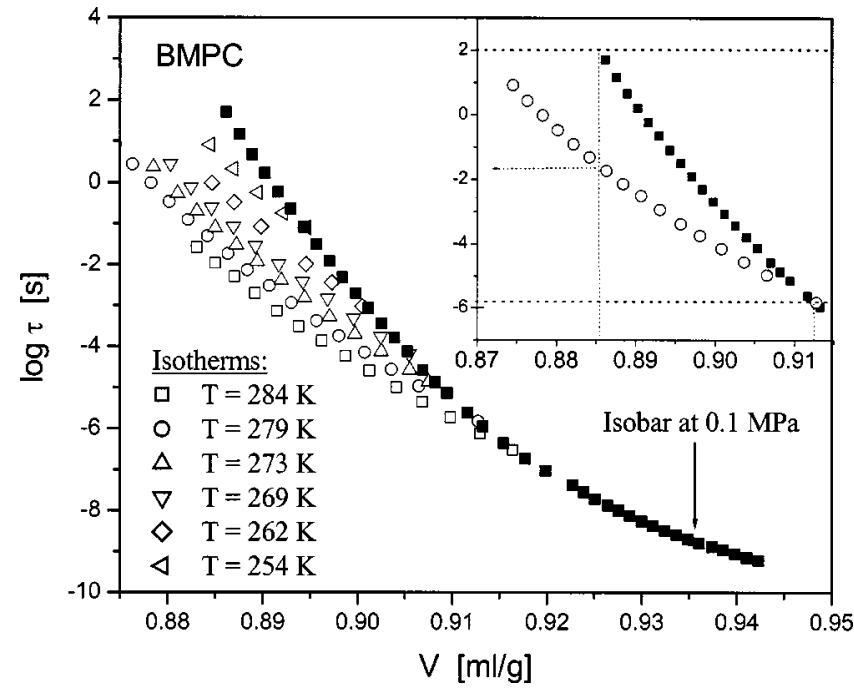

FIG. 3. The structural relaxation times of BMPC measured dielectrically (Ref. 29), as a function of the specific volume. The solid symbols are for atmospheric pressure and varying temperature, while the hollow symbols are varying pressure at the indicated temperatures. The inset shows on an expanded scale the region near the glass temperature for the isobar and one isotherm. Note that a change in volume at constant temperature causes more than half the change in relaxation time effected by the same volume change at constant pressure.

constant pressure (isobaric). ${ }^{31}$ The latter quantity is the activation enthalpy, more commonly referred to as the apparent activation energy

$$
E_{P}=\left.R \frac{\partial \ln \tau_{\alpha}}{\partial T^{-1}}\right|_{P} .
$$

We obtain $E_{P}$ for atmospheric pressure from the slope of the Arrhenius plot of $\tau_{\alpha}$, shown in Figs. 5 and 6 for BMPC and BMMPC, respectively. The corresponding isochoric activation energy,

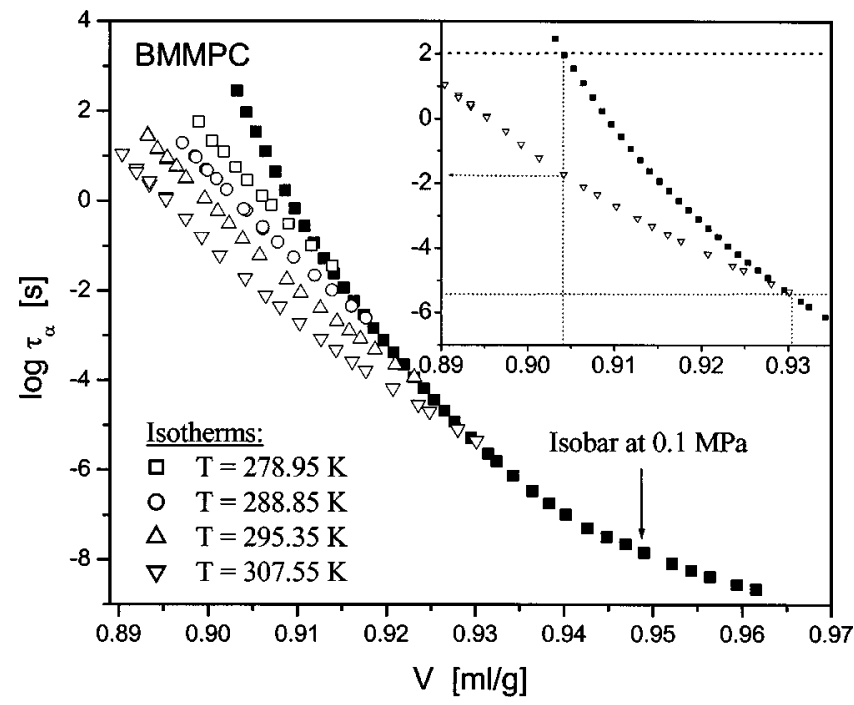

FIG. 4. The structural relaxation times of BMMPC measured dielectrically (Ref. 30), as a function of the specific volume, at the indicated temperatures. The inset shows on an expanded scale the region near the glass temperature for the isobar and one isotherm. Note that a change in volume at constant temperature causes roughly half the change in relaxation time effected by the same volume change at constant pressure.

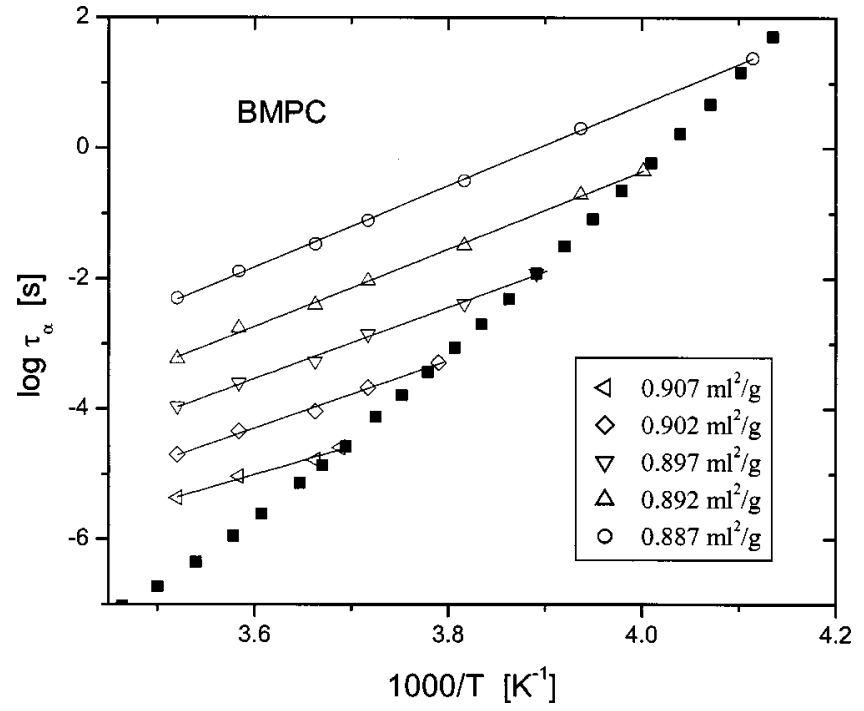

FIG. 5. Arrhenius plot of the structural relaxation times of BMPC measured at ambient pressure (solid symbols) and calculated for the indicated values of the specific volume (hollow symbols).

$$
E_{V}=\left.R \frac{\partial \ln \tau_{\alpha}}{\partial T^{-1}}\right|_{V},
$$

is extracted from Figs. 3 and 4 by determining $\tau_{\alpha}(T)$ for various fixed values of $V$. These isochoric relaxation times are included in Figs. 5 and 6 , with $E_{V}$ for $P=0.1 \mathrm{MPa}$ calculated from the slope at the intersection with the isobaric data.

The results are listed in Table II, revealing that $E_{V} / E_{P}$ $\sim 0.4$ for both liquids near $T_{g}$ at ambient pressure. The fact that this ratio is less than one-half indicates that volume exerts a somewhat greater influence on the relaxation times than does thermal energy, although both contributions are significant.

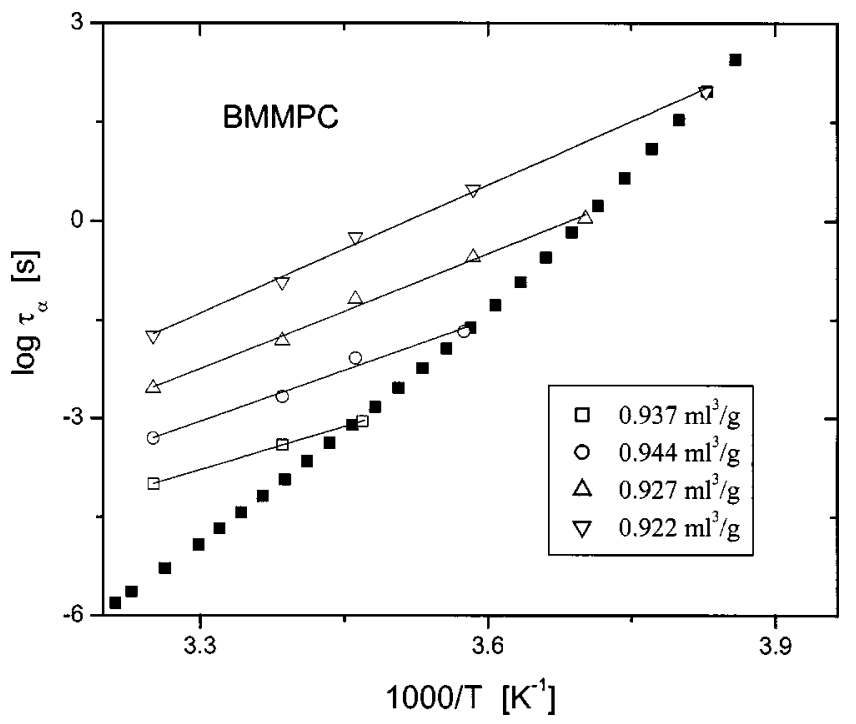

FIG. 6. Arrhenius plot of the structural relaxation times of BMMPC measured at ambient pressure (solid symbols) and calculated for the indicated values of the specific volume (hollow symbols). 
TABLE II. Isobaric and isochoric relaxation properties $\left(\tau_{\alpha}=1 \mathrm{~s}\right.$ and $\left.P=0.1 \mathrm{MPa}\right)$.

\begin{tabular}{ccccc}
\hline \hline & $\begin{array}{c}E_{P} \\
(\mathrm{MJ} / \mathrm{mol})\end{array}$ & $\begin{array}{c}E_{V} \\
(\mathrm{MJ} / \mathrm{mol})\end{array}$ & $\begin{array}{c}\alpha_{P} \\
\left(\mathrm{~K}^{-1}\right)\end{array}$ & $\begin{array}{c}\alpha_{\tau} \\
\left(\mathrm{K}^{-1}\right)\end{array}$ \\
\hline BMPC & $0.309 \pm 0.010$ & $0.119 \pm 0.010$ & $7.2 \pm 0.3 \times 10^{-4}$ & $-4.2 \pm 0.2 \times 10^{-4}$ \\
BMMPC & $0.290 \pm 0.008$ & $0.118 \pm 0.010$ & $5.4 \pm 0.3 \times 10^{-4}$ & $-3.9 \pm 0.1 \times 10^{-4}$ \\
\hline \hline
\end{tabular}

An alternative method of assessing the relative importance of temperature and volume is from the ratio of the isobaric expansion coefficient, $\alpha_{P}=-\rho^{-1}(\partial \rho / \partial T)_{P}$, to the coefficient of isochronal expansivity, $\alpha_{\tau}=-\rho^{-1}(\partial \rho / \partial T)_{\tau}$, the latter evaluated at a fixed value of the relaxation time. ${ }^{14}$ The $\alpha_{P}$ at $P=0.1 \mathrm{MPa}$ and the temperature at which $\tau_{\alpha}$ $=1 \mathrm{~s}$, obtained from the fit of Eq. (1) to the PVT measurements, are listed in Table II. For temperatures just above $T_{g}$, we can calculate the pressures for which $\tau_{\alpha}=1 \mathrm{~s}$, using the combined temperature and pressure dependences of the structural relaxation times for BMPC and BMMPC, as reported in Refs. 29 and 30, respectively. Equation (1) then yields the corresponding volumes, from which the isochronal expansion coefficient listed in Table II were calculated. These results yield $\left|\alpha_{\tau}\right| / \alpha_{P}=0.58 \pm 0.05$ for BMPC and $0.72 \pm 0.06$ for BMMPC. Ratios less than unity indicate that volume is the more important control parameter than temperature, ${ }^{14}$ consistent with the inference drawn from the activation energies.

\section{CONCLUSIONS}

Recently it has been suggested ${ }^{13,14}$ that at low pressures near $T_{g}$, temperature is the dominant control variable for structural relaxation of supercooled liquids and polymers. However, we have found that for various van der Waals liquids at low pressure, volume and thermal energy play a comparable role in governing the structural relaxation times. ${ }^{22,23,32,33}$ The only exception to this behavior appears to be strongly associated liquids, such as the polyalcohols. ${ }^{14,24}$ Evidently, the materials whose behavior led to the conclusion that temperature is dominant ${ }^{13,14}$ were not representative of all glass-formers. Moreover, for the two van der Waals liquids studied herein, we find that volume is actually more important than thermal energy. While this might be expected at sufficiently high densities, the stronger contribution of volume at atmospheric pressure is intriguing.

We can conclude that models for structural relaxation applicable to all classes of liquids cannot be based solely on thermally activated dynamics or on free volume concepts. More promising approaches include entropy theories, ${ }^{34,35}$ or energy landscape models in which the magnitudes of the potential barriers depend explicitly on the local density ${ }^{18}$ or, conversely, free volume models which include an energy barrier along with the volume requirements for motion. ${ }^{19,36}$ Of course, to the extent free volume does not scale in proportion to the measured volume, models based on the former are not ruled out by the fact that thermal energy does exert some effect on structural relaxation near $T_{g}$. Quantifying the unoccupied volume and its distribution in supercooled liquids, as can be accomplished with PALS, ${ }^{15-17,37,38}$ may prove decisive.

\section{ACKNOWLEDGMENTS}

The work at NRL was supported by the Office of Naval Research. Financial support of the Committee for Scientific Research, Poland (KBN, Grant Nos. 5PO3B 02220 and 5PO3B 14520) is gratefully acknowledged. We wish to thank A. Best for assistance with the PVT measurements

${ }^{1}$ M. Goldstein, J. Chem. Phys. 51, 3728 (1969).

${ }^{2}$ C. A. Angell, J. Non-Cryst. Solids 131-133, 13 (1991); Science 267, 1924 (1995); L. M. Martinez and C. A. Angell, Nature (London) 410, 663 (2001).

${ }^{3}$ F. H. Stillinger, Science 267, 1935 (1995); S. Sastry, P. G. Debenedetti, and F. H. Stillinger, Nature (London) 393, 554 (1998).

${ }^{4}$ M. Schulz, Phys. Rev. B 57, 11319 (1998).

${ }^{5}$ T. Keyes, Phys. Rev. E 59, 3207 (1999).

${ }^{6} \mathrm{~S}$. Glasstone, K. Laidler, and H. Eyring, The Theory of Rate Processes (McGraw-Hill, New York, 1941).

${ }^{7}$ J. Frenkel, Kinetic Theory of Liquids (Dover, New York, 1995).

${ }^{8}$ J. D. Ferry, Viscoelastic Properties of Polymers, 3rd ed. (Wiley, New York, 1980).

${ }^{9}$ M. L. Williams, R. F. Landel, and J. D. Ferry, J. Am. Chem. Soc. 77, 3701 (1955).

${ }^{10}$ M. H. Cohen and T. Turnbull, J. Chem. Phys. 31, 1164 (1959); D. Turnbull and M. H. Cohen, J. Chem. Phys. 34, 120 (1961); 52, 3038 (1970); M. H. Cohen and G. S. Grest, Phys. Rev. B 20, 1077 (1979).

${ }^{11}$ J. H. Hilderbrand, Science 174, 490 (1971).

${ }^{12}$ J. T. Bendler, J. J. Fontanella, and M. F. Shlesinger, Phys. Rev. Lett. 87, 195503 (2001).

${ }^{13}$ C. Alba-Siminoesco, D. Kivelson, and G. Tarjus, J. Chem. Phys. 116, 5033 (2002).

${ }^{14}$ M. L. Ferer, C. Lawrence, B. G. Demirjian, D. Kivelson, C. AlbaSimionesco, and G. Tarjus, J. Chem. Phys. 109, 8010 (1999).

${ }^{15}$ J. Bartos, O. Sausa, J. Kristiak, T. Blochowicz, and E. Rossler, J. Phys.: Condens. Matter 13, 11473 (2001).

${ }^{16}$ K. L. Ngai, L.-R. Bao, A. F. Yee, and C. L. Soles, Phys. Rev. Lett. 87, 215901 (2001).

${ }^{17}$ S. Vass, A. Patkowski, E. W. Fischer, K. Süvegh, and A. Vertes, Europhys. Lett. 46, 815 (1999).

${ }^{18}$ T. Pakula, J. Mol. Liq. 86, 109 (2000).

${ }^{19}$ P. B. Macedo and T. A. Litovitz, J. Chem. Phys. 42, 245 (1965).

${ }^{20}$ M. Paluch, C. M. Roland, and A. Best, J. Chem. Phys. 117, 1188 (2002).

${ }^{21}$ M. Paluch, R. Casalini, A. Best, and A. Patkowski, J. Chem. Phys. 117, 7624 (2002).

${ }^{22}$ M. Paluch, R. Casalini, and C. M. Roland, Phys. Rev. B 66, 092202 (2002).

${ }^{23}$ R. Casalini, M. Paluch, and C. M. Roland, J. Phys. Chem. B, submitted.

${ }^{24}$ S. Hensel-Bielowka, M. Paluch, and C. M. Roland, J. Phys. Chem. B 106, 12459 (2002)

${ }^{25}$ B. Gerharz, G. Meier, and E. W. Fischer, J. Chem. Phys. 92, 7110 (1990).

${ }^{26}$ P. Zoller and D. J. Walsh, Standard Pressure-Volume Temperature Data for Polymers (Technomic, Lancaster, 1995).

${ }^{27}$ G. Meier, B. Gerharz, and D. Boese, J. Non-Cryst. Solids 131-133, 144 (1991).

${ }^{28}$ P. Zoller, in Polymer Handbook, 3rd ed., edited by J. Brandrup and H. Immergut (Wiley, New York, 1989).

${ }^{29}$ S. Hensel-Bielowka, J. Ziolo, M. Paluch, and C. M. Roland, J. Chem. Phys. 117, 2317 (2002).

${ }^{30}$ R. Casalini, M. Paluch, and C. M. Roland, Phys. Rev. E (to be published).

${ }^{31}$ M. Naoki, H. Endou, and K. Matsumoto, J. Phys. Chem. 91, 4169 (1987).

${ }^{32}$ M. Paluch, C. M. Roland, J. Gapinski, and A. Patkowski, J. Chem. Phys. (to be published).

${ }^{33}$ C. M. Roland and R. Casalini, Macromolecules (to be published). 
${ }^{34}$ I. Avramov, J. Non-Cryst. Solids 262, 258 (2000).

${ }^{35}$ R. Casalini, S. Capaccioli, M. Lucchesi, P. A. Rolla, and S. Corezzi, Phys. Rev. E 63, 031207 (2001); R. Casalini, M. Paluch, J. J. Fontanella, and C. M. Roland, J. Chem. Phys. 117, 4901 (2002).

${ }^{36}$ G. C. Berry and T. G. Fox, Adv. Polym. Sci. 5, 261 (1967).
${ }^{37}$ G. Dlubek, D. Bamford, A. Rodriguez-Gonzalez, S. Bornemann, J. Stejny, B. Schade, M. A. Alam, and M. Arnold, J. Polym. Sci., Polym. Phys. Ed. 40, 434 (2002).

${ }^{38}$ B. Wang, Z. F. Wang, M. Zhang, W. H. Liu, and S. J. Wang, Macromolecules 35, 3993 (2002). 